\title{
The role of perioperative radiotherapy in gastric cancer
}

\author{
Thales Paulo Batista, ${ }^{1}$ Lucas Marques de Mendonça, ${ }^{2}$ Ana Luiza Fassizoli-Fonte ${ }^{2}$ \\ ${ }^{1}$ Department of Surgery/Oncology; ${ }^{2}$ Department of Radiotherapy, FPS/IMIP - Faculdade Pernambu- \\ cana de Saúde, Instituto de Medicina Integral Professor Fernando Figueira, Recife/PE, Brazil
}

\begin{abstract}
Gastric cancer is one of the most common neoplasms and a main cause of cancer-related mortality worldwide. Surgery remains the mainstay for cure and is considered for all patients with potentially curable disease. However, despite the fact that surgery alone usually leads to favorable outcomes in early stage disease, late diagnosis usually means a poor prognosis. In these settings, multimodal therapy has become the established treatment for locally advanced tumors, while the high risk of locoregional relapse has favored the inclusion of radiotherapy in the comprehensive therapeutic strategy. We provide a critical, non-systematic review of gastric cancer and discuss the role of perioperative radiation therapy in its treatment.
\end{abstract}

\section{Introduction}

Gastric cancer is one of the main causes of cancer-related death worldwide and most patients are often diagnosed at locally advanced stages. ${ }^{1,2}$ In these cases, radical surgery remains the mainstay for cure. However, the elevated rates of recurrence mean that late stage gastric cancer is difficult to cure by surgery alone. ${ }^{3-7}$ Therefore, combination therapy has become an established treatment, whereas the high risk of locoregional relapse has favored the inclusion of radiotherapy as a perioperative component of its comprehensive therapeutic strategy.

Correspondence: Thales Paulo Batista, Rua Gois Cavalvante, 100, apt. 1206, Tamarineira, Recife/PE, 52060-140, Brazil. Tel. +55.81.8886.1203.

E-mail: t.paulo@bol.com.br

Key words: stomach cancer, radiation therapy, multimodal treatments.

Contributions: the authors contributed equally.

Conflict of interests: the authors declare no potential conflict of interests.

Received for publication: 17 October 2012.

Revision received: 3 December 2012.

Accepted for publication: 6 December 2012.

This work is licensed under a Creative Commons Attribution NonCommercial 3.0 License (CC BY-NC 3.0).

(C) Copyright T.P. Batista et al., 2012

Licensee PAGEPress, Italy

Oncology Reviews 2012; 6:e23

doi:10.4081/oncol.2012.e23

\section{Radiation as single modality}

Radiotherapy alone as adjuvant treatment was first explored in 1989 by the British Stomach Cancer Group. ${ }^{8}$ In that trial, gastric cancer patients from 10 centers throughout the United Kingdom were randomly allocated to receive surgery alone, surgery plus postoperative chemotherapy (mitomycin C, adriamycin and 5-FU), or postoperative radiotherapy with a defined dose of $45 \mathrm{~Gy}$. No survival advantage was shown for those patients receiving either adjuvant therapy compared to those undergoing surgery alone after at least five years follow up. ${ }^{9}$ Similarly, no benefit for intraoperative radiotherapy (i.e., single application of 28 Gy to the celiac axis) was seen in a German randomized trial conduced by Krämling et al. ${ }^{10}$

In 1998, Zhang et al. ${ }^{11}$ also initially evaluated the use of preoperative radiotherapy alone in Chinese patients with adenocarcinoma of the gastric cardia. In their prospective randomized trial, the authors reported increased resection rates and downstaging favoring the neoadjuvant radiotherapy. Operative mortality rates were reported to be $0.6 \%$ versus $2.5 \%$ with 10 -year survival rates of $20.26 \%$ versus $13.30 \%$ for preoperative radiotherapy versus surgery alone, respectively. In contrast, two Russian studies published by Skoropad et $a l .{ }^{12,13}$ reported that neoadjuvant radiotherapy seems not to be sufficient to improve survival in gastric cancer patients. However, the results were promising in selected subgroups of patients treated with a combined preoperative plus intraoperative radiation protocol. A marked survival advantage was seen in subset analysis of lymphnode involvement $(\mathrm{P}=0.04)$ and extragastric tumor extension $(\mathrm{P}=0.042) .{ }^{12}$ In both trials, neoadjuvant radiotherapy was described as well tolerated and it has, therefore, been considered an important area of research for future refinement of adjuvant treatment in North American settings. The main results of these trials are summarized in Table 1.

\section{Postoperative chemoradiation}

In order to increase the effectiveness of radiation on tumor cells and, therefore, improve outcomes, radiotherapy has mainly been used in combined chemoradiation regimens. The Mayo Clinic performed the first trial to present significant results favoring the use of postoperative chemoradiotherapy versus surgery alone. ${ }^{14}$ This study enrolled 62 patients and local control was achieved in $61 \%$ of patients treated with adjuvant chemoradiation versus $45 \%$ in the surgery-alone group, while the 5 -year survival also favored the adjuvant therapy group (20\% vs 4\%). However, this study has been criticized because randomization took place before patient consent for the study had been obtained, and $25 \%$ of patients refused treatment. The patients who refused treatment actually had the best survival of all groups (30\% of 5 -year sur- 
vival). Furthermore, there was a high rate of discontinuation of treatment due to local side effects from radiotherapy.

Chemoradiotherapy had been occasionally used in the treatment of gastric cancer up to $2001,{ }^{15}$ when the results of the INT-0116 trial showed an improvement in disease-free (DFS) and overall (OS) survival by chemoradiation with a significant impact on tumor management. ${ }^{3}$ Based on these data, there has been an increasing interest in radiotherapy and its association with chemotherapy for patients with locoregional disease as a part of adjuvant treatment after surgery in high-risk patients. ${ }^{15,16}$

Major changes in adjuvant therapy for gastric cancer favoring chemoradiation followed the results of the INT-0116 trial. ${ }^{3}$ Since publication of this study, in the USA, the use of postoperative chemoradiation in stomach cancer has been considered a standard of care. ${ }^{17}$ Its original regimen utilized two cycles of 5-FU and leucovorin followed by concurrent chemoradiotherapy with $45 \mathrm{~Gy}$ of radiation administered using fields encompassing the entire gastric bed, regional nodes, and $2 \mathrm{~cm}$ proximal and distal margin. As far as the efficacy of combined therapy is concerned, this study demonstrated that patients treated with postoperative radiochemotherapy showed significantly better 3 -year $\mathrm{OS}(50 \%$ vs $41 \% ; \mathrm{P}=0.005)$ as well as 3 -year DFS survival (48 vs 31\%; $\mathrm{P}=0.001$ ) than those who were only treated with surgery. ${ }^{3}$ After over ten years of follow up, chemoradiation demonstrates continued benefit in terms of OS and DFS, and hazard ratios remain virtually unchanged since the original report. The authors also pointed out that subset analyses show robust treatment benefit in most subsets, with the exception of patients with diffuse histology. ${ }^{18}$ Nevertheless, the biggest benefit from chemoradiation was seen in those patients with higher $\mathrm{N}$ stages and limited lymph node dissection.

The main criticisms of the INT-0116 trial relate to the high toxicity of this regimen and the low quality of the surgery performed. In this study, $41 \%$ of patients had grade 3 toxicity and $32 \%$ had grade 4 , while $31 \%$ did not complete treatment due to toxicity. Furthermore, the other main weakness of this trial was that only $10 \%$ of patients included in the analysis had undergone a formal D2-lymphadenectomy. A D1-dissection had been performed in $36 \%$, but most patients (54\%) had undergone a D0-dissection, which is less than a complete dissection of the N1 nodes. ${ }^{3}$ Therefore, some questions remain unanswered about the toxicity of this approach and its efficacy after adequate surgery. ${ }^{15,19}$

Whether chemoradiation may contribute to improve outcomes after a D2-dissection was initially explored in two observational Asian studies. ${ }^{20,21}$ In a Korean study, 544 patients received postoperative chemoradiation according to the INT-0116 protocol after curative D2resection, and the results were compared with those of 446 patients who received surgery alone. The results showed a significantly prolonged median OS (95.6 vs 62.6 months; HR 0.80; $\mathrm{P}=0.02$ ) and DFS sur- vival (75.6 vs 52.7 months; HR $0.80 ; \mathrm{P}=0.016$ ) in the chemoradiation group. Three-year survival was $60 \%$ in the group treated with adjuvant chemoradiation..$^{20}$ Accordingly, in a cohort study of 70 patients who received D2-dissection and adjuvant chemoradiation, Leong et al. ${ }^{21}$ reported 3 -year OS, DFS, and local control of $60.6 \%, 54.1 \%$, and $84.3 \%$, respectively. However, as pointed out by Kwon et al. ${ }^{22}$ different patient groups and radiation techniques to from those in the INT-0116 study were used. Also, all patients presented pathological stage T3-4 or nodepositive disease, and a significant number of patients were treated with 3D conformal radiation.

Initially, a clinical trial conducted by Kwon and colleagues ${ }^{22}$ tried to evaluate the role of applying an adjuvant chemoradiotherapy regimen in the context of D2-dissection. Nevertheless, no conclusion could be drawn about the benefit of adding radiation to adjuvant chemotherapy because of the small sample size; this was due to early closure. The crude rate of locoregional relapse of the chemoradiation arm in this study was $12.9 \%$, which was consistent with the results of the Korean ${ }^{20}$ and Singaporean ${ }^{21}$ studies previously cited (13\% and $15 \%$, respectively). Five-year DFS and OS were $70.1 \%$ and $76.2 \%$, respectively, among patients treated with chemoradiation, but there was no statistically significant difference between the two groups. Additionally, these authors found no differences in clinical outcomes between the chemoradiation and chemotherapy treatment groups. Loco-regional failure tended to be less frequent in the chemoradiation group ( $12.9 \%$ vs $23.3 \%, \mathrm{P}=0.335)$, but grade 3 and 4 neutropenia was also more common $(48.4 \%$ vs $16.7 \%)^{22}$

Later, the Adjuvant Chemoradiation Therapy in Stomach Cancer (ARTIST) trial investigated the efficacy of postoperative chemoradiation as adjuvant treatment using a capecitabine plus cisplatin regimen in patients with gastric cancer who underwent curative D2-gastrectomy. ${ }^{23}$ After a median follow up of 53.2 months, the estimated 3 -year DFS rates were $78.2 \%$ in the chemoradiation arm and $74.2 \%$ in the chemotherapy arm. Although there was no significant difference in DFS between the two treatment arms $(\mathrm{P}=0.0862)$, there was a statistically significant prolongation favoring the chemoradiation arm in positive pathological lymph node $(\mathrm{P}=0.0365)$ and the multivariate analysis demonstrated significantly increased DFS after adjustment for subgroup stages IB and II versus III and IV (HR 0.68; 95\% CI: 0.473-0.995). Because these findings were from a subgroup analysis, the authors suggested these data should be interpreted with caution and planned a subsequent phase III trial (ARTIST-II) to compare chemotherapy versus chemoradiation in patients with D2-lymphadenectomy and pathological lymph node-positive disease in order to confirm the benefit of adjuvant chemoradiation.

In the ARTIST trial, treatment was completed as planned by $75.4 \%$ of

Table 1. Summary of results from main trials exploring radiation as single modality.

\begin{tabular}{|c|c|c|c|c|c|}
\hline Trials & Year & Treatment arms & No. & 5-year survival (\%) & Comments \\
\hline British Stomach Cancer Group ${ }^{8,9}$ & $1989 / 94$ & $\begin{array}{c}\text { Surgery only } \\
\text { External adjuvant RT } \\
\text { Adjuvant chemoterapy }\end{array}$ & $\begin{array}{l}145 \\
153 \\
138\end{array}$ & $\begin{array}{l}20 \\
12 \\
19\end{array}$ & $\mathrm{NS}(\mathrm{P}=0.14)$ \\
\hline Krämling et al..$^{10}$ & 1996 & $\begin{array}{c}\text { Surgery only } \\
\text { Intraoperative RT }\end{array}$ & $\begin{array}{l}64 \\
51\end{array}$ & $\begin{array}{l}\text { NR } \\
\text { NR }\end{array}$ & Mean survival 30.8 vs 26.9 months (NS) \\
\hline Zhang et al. ${ }^{11}$ & 1998 & $\begin{array}{c}\text { Surgery only } \\
\text { Neoadjuvant RT }\end{array}$ & $\begin{array}{l}199 \\
171\end{array}$ & $\begin{array}{l}20.3 \\
30.1\end{array}$ & $\begin{array}{l}\text { Tumors from gastric cardia } \\
\text { 10-year survival of } 13.3 \% \\
\text { vs. } 19.7 \% ;(\mathrm{P}=0.009)\end{array}$ \\
\hline Skoropad et al. ${ }^{12}$ & 2000 & $\begin{array}{c}\text { Surgery only } \\
\text { Neoadjuvant and operative RT }\end{array}$ & $\begin{array}{l}53 \\
59\end{array}$ & $\begin{array}{l}43 \\
44\end{array}$ & Estimated from survival curve \\
\hline Skoropad et al. ${ }^{13}$ & 2002 & $\begin{array}{c}\text { Surgery only } \\
\text { Neoadjuvant RT }\end{array}$ & $\begin{array}{l}75 \\
77\end{array}$ & $\begin{array}{l}30 \\
39\end{array}$ & 10 -year survival of $32 \%$ us $18 \%$; NS ( $\mathrm{P}=0.55)$ \\
\hline
\end{tabular}

RT, radiotherapy; NR, not reported; NS, not significant. 
patients in the chemotherapy arm versus $81.7 \%$ in the chemoradiation arm. Adverse events that led to treatment modifications (delays or dose reductions) occurred in $52 \%$ versus $35 \%$ of patients..$^{23}$ In contrast, the study conduced by Kwon et al. ${ }^{22}$ utilized a protocol almost identical to the ARTIST trial, with the exception of using capecitabine/cisplatin rather than 5-FU/cisplatin as systemic chemotherapy. However, $27 \%$ of their patients were unable to complete all planned therapies due to toxicity, and the overall rates of grade 3-4 neutropenia in this study were $48.4 \%$. These findings suggest that an adjuvant regimen employing 5FU/cisplatin as the systemic component prior to and after concurrent chemoradiation with oral capecitabine is unacceptably toxic.

Very recently, Zhu et al. ${ }^{24}$ presented their phase III trial comparing postoperative intensity-modulated radiotherapy plus chemotherapy (IMRT-C) with chemotherapy only after a curative D2-dissection. Using a 5-FU/leucovorin chemotherapy regimen and a 45 Gy of IMRT radiation, grades 3 and 4 toxicities occurred in a small proportion of patients in both arms. There was no significant difference in 5-year OS between groups ( $48.4 \%$ vs $41.8 \% ; \mathrm{P}=0.122$ ), but the chemoradiation arm experienced increased DFS (32 vs 50 months). While different forms of adjuvant chemotherapy are standards in Asia, a major criticism of this study is that more effective cytotoxic regimens are currently available for treatment of gastric cancer patients.

The benefit of adding radiotherapy to adjuvant chemotherapy was also explored in phase III trials by Bamias et al. ${ }^{25}$ and Kim et al. ${ }^{26}$ The former observed that neither local recurrence rates (10\% vs $5 \%)$ nor 3-year OS or DFS were significantly improved by addition of radiation to platinum/docetaxel chemotherapy ( $61 \%$ vs $57 \%$ and $51 \%$ vs $48 \%$, respectively). ${ }^{25}$ Applying a chemoradiation regimen basically identical to those used in the INT-0116 protocol for stage III and IV(M0) patients, Kim and colleagues $^{26}$ observed that addition of radiation to the 5-FU/leucovorin regimen significantly improved locoregional recurrence-free survival, but not DFS. Unfortunately, both trials were terminated early.

Reporting their 10-year experience with adjuvante chemoradiation for resected gastric cancer, Chang et al. ${ }^{27}$ found that radiotherapy techniques were important prognostic factors, emphasizing the need for more sophisticated radiotherapy techniques to optimize the therapeutic ratio. These authors also emphasized that $35 \%$ of the radiotherapy planned in the INT-0116 study deviated from the protocol, reflecting the inadequacies of conventional techniques in targeting delineation. Equally, Leong and colleagues pointed out that $35 \%$ of radiotherapy treatment plans contained protocol deviations that could have been satisfactorily amended before commencement of treatment by using pre- cisely defined, multiple-field, 3-D conformal techniques. ${ }^{28}$ In spite of this, even with conformal radiotherapy, chemoradiation regimens have also been associated with significant toxicity, ${ }^{22,29}$ which is possibly influenced by the different chemotherapies delivered. ${ }^{22}$

Attempts to improve the chemoradiation regimens were recently reported in the CALGB 80101 trial that combined modern regimens of perioperative chemoterapy with fluoropyrimidine-based radiotherapy. Preliminary results of this study were presented at the 2011 American Society of Clinical Oncology (ASCO) annual meeting. ${ }^{30}$ Accordingly, the authors reported that postoperative chemoradiation using ECF before and after 5-FU/radiation did not improve survival when compared to bolus 5FU/leucovorin before and after 5-FU/radiation. Thus, the original INT0116 5-FU/leucovorin regimen remains standard for adjuvant chemoradiation. Results from the main phase III trials exploring chemoradiation approaches for gastric cancer are summarized in Table 2.

Finally, despite the fact that perioperative chemotherapy (neoadjuvant and adjuvant) is an estabilished treatment for locally advanced tumors in many European countries, the ongoing CRITICS $^{31}$ trial is currently open to recruitment in The Netherlands. This study aims to evaluate the theoretical advantages of adding radiotherapy to this approach. Since locoregional failure rates in patients with locally advanced resectable gastric cancer are quite high, it seems that the results of this trial will strengthen the need for radiation therapy as an integral part of their treatment. ${ }^{32}$ Its multicenter randomized phase III protocol investigates whether chemoradiotherapy (45 Gy in 5 weeks with daily cisplatin and capecitabine) after preoperative chemotherapy ( $3 \times$ epirubicin, cisplatin, capecitabine, ECC) and adequate (D1+) surgery leads to improved survival in comparison with postoperative chemotherapy $(3 \times \mathrm{ECC})$.

\section{Preoperative chemoradiation}

Neoadjuvant treatments have received increasing attention in the current multidisciplinary treatment model of locally advanced gastric cancer, especially since this approach may improve patient tolerance given that surgery-related morbidity and symptoms usually have a negative impact on performance status. In addition, the preoperative approach can result in a downstaging of the tumors that consequently might improve the curative resection rate in patients with advanced $\mathrm{T}$ and $\mathrm{N}$ stage disease.

Table 2. Summary of results from main phase III trials of chemoradiation therapy in $\mathbf{5 4 6}$ gastric cancer patients.

\begin{tabular}{|c|c|c|c|c|c|c|}
\hline \multirow[t]{2}{*}{ Trials } & \multirow[t]{2}{*}{ No. } & \multirow[t]{2}{*}{ HR for OS (95\% CI) } & \multirow[t]{2}{*}{ HR for DFS ( $95 \%$ CI) } & \multicolumn{2}{|c|}{ Major toxicities (\%) } & \multirow[t]{2}{*}{ Comments } \\
\hline & & & & Death & penia ( $\geq \operatorname{gr} 3$ ) & \\
\hline INT-0116 $6^{3,18}$ & & $1.32(1.10-1.60)$ & $1.51(1.25-1.83)$ & & & Recently up-dated \\
\hline 5-FU/LV/RT & 281 & & & 1 & 54 & \\
\hline Surgery only & 275 & & & - & - & \\
\hline ARTIST ${ }^{23}$ & & NR & NR & & & 3-year DFS $78.2 \%$ vs $74.2 \%$ \\
\hline XP/XRT/XP & 230 & & & 0.4 & 48.4 & \\
\hline XP & 228 & & & 0.4 & 40.7 & \\
\hline Zhu et al. ${ }^{24}$ & & $1.24(0.94-1.65)$ & $1.35(1.03-1.78)$ & & & IMRT \\
\hline 5-FU/LV/RT & 186 & & & 0 & 7.5 & \\
\hline 5-FU/LV & 165 & & & 0 & 7.3 & \\
\hline CALGB $80101^{30}$ & & $1.03(0.80-1.34)$ & $1.00(0.79-1.27)$ & & & Not published in full \\
\hline 5-FU/LV/RT & NR* & & & 3 & 53 & \\
\hline ECF/RT & NR & & & $<1$ & 48 & \\
\hline
\end{tabular}

HR, hazard ratio; OS, overall survival; DFS, disease-freee survival; CI, confidence interval; NR, not reported; XP, capecitabine plus cisplatin; XP/XRT/XP, capecitabine plus cisplatin and radiotherapy with capecitabine; ECF/RT, epirubicin, cisplatin, and infusional 5-FU before and after 5-FU/radiotherapy; 5-FU/LV/RT, 5-FU plus leucovorin and radiotherapy; IMRT, intensity-modulated radiation therapy. *Total of 546 patients. 
Unfortunately, there is currently no phase III study supporting a purely neoadjuvant strategy of chemoradiation for operable gastric cancer. Stahl et al. ${ }^{33}$ tried to address the important concern of whether preoperative versus postoperative chemoradiation improves patient survival in gastric cancer; but their trial was prematurely stopped due to insufficient accrual and failed to provide a definitive anwer. The authors in this trial randomly allocated patients with locally advanced (uT3-4NXM0) adenocarcinoma of the esophagogastric junction to induction chemotherapy followed by surgery or chemotherapy followed by chemoradiotherapy plus surgery. There was no difference in the number of patients undergoing complete tumor resection between treatment groups ( $69.5 \%$ vs $71.5 \%)$; however, patients in the chemoradiation arm had a significantly higher probability of showing pathological complete response ( $15.6 \%$ vs $2.0 \%)$ or tumor-free lymph nodes (64.4\% vs $37.7 \%)$ at resection. Also, there was no significant increase in postoperative mortality in the chemoradiotherapy group $(10.2 \% \mathrm{vs}$ $3.8 \%$; $\mathrm{P}=0.26$ ), which improved 3 -year survival rate from $27.7 \%$ to $47.4 \%$ ( $\mathrm{P}=0.07)$. Similarly, the design of the ongoing Treatment of Gastric Cancer with Chemotherapy and Chemoradiotherapy trial (TRACE) has the potential to verify the theoretical advantages of induction chemoradiation over the postoperative approach (NCT00512304).

Most of the available data concerning preoperative chemoradiation are currently coming from institutional phase II trials. In these settings, the experience from the MD Anderson Cancer Center has demonstrated that this comprehensive treatment is well tolerated and leads to appropriate pathological outcomes. ${ }^{34,35}$ The results of these trials are important in the design of future prospective studies and open up interesting new pathways to upcoming protocols for the use of preoperative chemoradiation in gastric cancer.

\section{Final comments}

The policy of surgery alone for locally advanced gastric cancer has been abandoned, but an optimal multimodal and comprehensive therapeutic strategy has not yet been established. Efforts should, therefore, be made to accurately select patients to receive a combined treatment approach. Given this, the current medical literature has mainly supported the use of chemoradiation as an IMRT technique after curative resection in patients with positive lymph node status, postoperative residual tumor or close/positive margins, more advanced stages, and limited lymph node dissection (D0 and D1), especially for those who did not receive neoadjuvant chemotherapy. ${ }^{23,27,32,36-41}$ In these settings, the ratio between metastatic and examined lymph nodes (N-ratio) ${ }^{42-46}$ might improve patient selection after an appropriated D2-gastrectomy, ${ }^{47}$ whereas the benefit of chemoradiation appeared to increase in line with higher $\mathrm{N}$ stages. ${ }^{18}$ Furthermore, combined chemoradiation may be considered for patients with initially unresectable gastric cancer. A possible way to improve the efficacy of adjuvant chemoradiation would be to integrate modern radiotherapy techniques with newer chemotherapy agents.

\section{References}

1. Jemal A, Bray F, Center MM, et al. Global cancer statistics. CA Cancer J Clin 2011;61:69-90.

2. Everett SM, Axon AT. Early gastric cancer in Europe. Gut 1997;41:142-50.

3. Macdonald JS, Smalley SR, Benedetti J, et al. Chemoradiotherapy after surgery compared with surgery alone for adenocarcinoma of the stomach or gastroesophageal junction. New Engl J Med 2001;345:725-30.

4. Cunningham D, Allum WH, Stenning SP, et al. Perioperative chemotherapy versus surgery alone for resectable gastroesophageal cancer. New Engl J Med 2006;355:11-20.

5. Ychou M, Boige V, Pignon JP, et al. Perioperative chemotherapy compared with surgery alone for resectable gastroesophageal adenocarcinoma: an FNCLCC and FFCD multicenter phase III trial. J Clin Oncol 2011;29:1715-21.

6. Sasako M, Sakuramoto S, Katai H, et al. Five-year outcomes of a randomized phase III trial comparing adjuvant chemotherapy with S-1 versus surgery alone in stage II or III gastric cancer. J Clin Oncol 2011;29:4387-93.

7. Bang YJ, Kim YW, Yang HK, et al. Adjuvant capecitabine and oxaliplatin for gastric cancer after D2 gastrectomy (CLASSIC): a phase 3 open-label, randomised controlled trial. Lancet 2012;379:315-21.

8. Allum WH, Hallissey MT, Ward LC, et al. A controlled, prospective, randomised trial of adjuvant chemotherapy or radiotherapy in resectable gastric cancer: interim report. British Stomach Cancer Group. Br J Cancer 1989;60:739-44.

9. Hallissey MT, Dunn JA, Ward LC, et al. The second British Stomach Cancer Group trial of adjuvant radiotherapy or chemotherapy in resectable gastric cancer: five-year follow-up. Lancet 1994;343:1309-12.

10. Krämling HJ, Wilkowski R, Dühmke E, et al. [Adjuvant intraoperative radiotherapy of stomach carcinoma]. [Article in German]. Langenbecks Arch Chir Suppl Kongressbd 1996;113:211-3.

11. Zhang ZX, Gu XZ, Yin WB, et al. Randomized clinical trial on the combination of preoperative irradiation and surgery in the treatment of adenocarcinoma of gastric cardia (AGC)--report on 370 patients. Int J Radiat Oncol 1998;42:929-34.

12. Skoropad VY, Berdov BA, Mardynski YS, et al. A prospective, randomized trial of pre-operative and intraoperative radiotherapy versus surgery alone in resectable gastric cancer. Eur J Surg Oncol 2000;26:773-9.

13. Skoropad V, Berdov B, Zagrebin V. Concentrated preoperative radiotherapy for resectable gastric cancer: 20 -years follow-up of a randomized trial. J Surg Oncol 2002;80:72-8.

14. Moertel CG, Childs DS, O'Fallon JR, et al. Combined 5-fluorouracil and radiation therapy as a surgical adjuvant for poor prognosis gastric carcinoma. J Clin Oncol 1984;2:1249-54.

15. Orditura M, Martinelli E, Galizia G, et al. Chemoradiotherapy as adjuvant treatment of gastric cancer. Ann Oncol 2007; 6 (Suppl. 18):vi133-5.

16. Kozak KR, Moody JS. The survival impact of the intergroup 0116 trial on patients with gastric cancer. Int $\mathrm{J}$ Radiat Oncol 2008;72:517-21.

17. NCCN. NCCN Clinical Practice Guidelines in Oncology. Gastric Cancer. Version 2.2011. 2011. Available from: http://www.nccn.org Accessed: December 5, 2011.

18. Smalley SR, Benedetti JK, Haller DG, et al. Updated Analysis of SWOG-Directed Intergroup Study 0116: A Phase III Trial of Adjuvant Radiochemotherapy Versus Observation After Curative Gastric Cancer Resection. J Clin Oncol 2012;30:2327-33.

19. Batista TP, Martins MR. Lymph node dissection for gastric cancer: a critical review. Oncol Rev 2012;6:e12.

20. Kim S, Lim DH, Lee J, et al. An observational study suggesting clinical benefit for adjuvant postoperative chemoradiation in a population of over 500 cases after gastric resection with D2 nodal dissection for adenocarcinoma of the stomach. Int $\mathrm{J}$ Radiat Oncol 2005;63:1279-85.

21. Leong CN, Chung HT, Lee KM, et al. Outcomes of adjuvant chemoradiotherapy after a radical gastrectomy and a D2 node dissection for gastric adenocarcinoma. Cancer J 2008;14:269-75. 
22. Kwon HC, Kim MC, Kim KH, et al. Adjuvant chemoradiation versus chemotherapy in completely resected advanced gastric cancer with D2 nodal dissection. Asia Pac J Clin Oncol 2010;6:278-85.

23. Lee J, Lim do H, Kim S, et al. Phase III trial comparing capecitabine plus cisplatin versus capecitabine plus cisplatin with concurrent capecitabine radiotherapy in completely resected gastric cancer with D2 lymph node dissection: the ARTIST trial. J Clin Oncol 2012;30:268-73.

24. Zhu WG, Xua DF, Pu J, et al. A randomized, controlled, multicenter study comparing intensity-modulated radiotherapy plus concurrent chemotherapy with chemotherapy alone in gastric cancer patients with D2 resection. Radiother Oncol 2012;104:361-6.

25. Bamias A, Karina M, Papakostas P, et al. A randomized phase III study of adjuvant platinum/docetaxel chemotherapy with or without radiation therapy in patients with gastric cancer. Cancer Chemoth Pharm 2010;65:1009-21.

26. Kim TH, Park SR, Ryu KW, et al. Phase 3 Trial of postoperative chemotherapy alone versus chemoradiation therapy in stage III-IV gastric cancer treated with R0 gastrectomy and D2 lymph node dissection. Int J Radiat Oncol 2012;84:e582-92.

27. Chang AT, Ng WT, Law AL, et al. Adjuvant chemoradiation for resected gastric cancer: a 10-year experience. Gastric Cancer 2011;14:63-71.

28. Leong T, Joon DL, Willis D, et al. Adjuvant chemoradiation for gastric cancer using epirubicin, cisplatin, and 5-fluorouracil before and after three-dimensional conformal radiotherapy with concurrent infusional 5-fluorouracil: a multicenter study of the Trans-Tasman Radiation Oncology Group. Int J Radiat Oncol 2011;79:690-5.

29. Kassam Z, Lockwood G, O'Brien C, et al. Conformal radiotherapy in the adjuvant treatment of gastric cancer: Review of 82 cases. Int J Radiat Oncol 2006;65:713-9.

30. Fuchs CS, Tepper JE, Niedzwiecki D, et al. Postoperative adjuvant chemoradiation for gastric or gastroesophageal junction (GEJ) adenocarcinoma using epirubicin, cisplatin, and infusional (CI) 5FU (ECF) before and after CI 5-FU and radiotherapy (CRT) compared with bolus 5-FU/LV before and after CRT: Intergroup trial CALGB 80101. J Clin Oncol 2011;29 (Suppl.):4003 (abstr.).

31. Dikken JL, van Sandick JW, Maurits Swellengrebel HA, et al. Neoadjuvant chemotherapy followed by surgery and chemotherapy or by surgery and chemoradiotherapy for patients with resectable gastric cancer (CRITICS). BMC Cancer 2011;11:329.

32. Koukourakis GV. Evidence based radiation therapy for locally advanced resectable and unresectable gastric cancer. World $\mathrm{J}$ Gastrointest Oncol 2011;3:131-6.

33. Stahl M, Walz MK, Stuschke M, et al. Phase III comparison of preoperative chemotherapy compared with chemoradiotherapy in patients with locally advanced adenocarcinoma of the esophagogastric junction. J Clin Oncol 2009;27:851-6.
34. Ajani JA, Winter K, Okawara GS, et al. Phase II trial of preoperative chemoradiation in patients with localized gastric adenocarcinoma (RTOG 9904): quality of combined modality therapy and pathologic response. J Clin Oncol 2006;24:3953-8.

35. Chakravarty T, Crane $\mathrm{CH}$, Ajani JA, et al. Intensity-modulated radiation therapy with concurrent chemotherapy as preoperative treatment for localized gastric adenocarcinoma. Int $\mathrm{J}$ Radiat Oncol 2012;83:581-6.

36. Kantzou I, Sarris G, Poulizi M, et al. Gastric cancer and adjuvant chemoradiotherapy: when and where, that's the question. J BUON 2011;16:473-7.

37. Kundel Y, Purim 0, Idelevich E, et al. Postoperative chemoradiation for resected gastric cancer - is the Macdonald Regimen Tolerable? a retrospective multi-institutional study. Radiat Oncol 2011;6:127.

38. Orditura M, De Vita F, Muto P, et al. Adjuvant chemoradiotherapy in patients with stage III or IV radically resected gastric cancer: a pilot study. Arch Surg 2010;145:233-8.

39. Kofoed SC, Muhic A, Baeksgaard L, et al. Survival after adjuvant chemoradiotherapy or surgery alone in resectable adenocarcinoma at the gastro-esophageal junction. Scand J Surg 2012;101:26-31.

40. Dikken JL, Jansen EP, Cats A, et al. Impact of the extent of surgery and postoperative chemoradiotherapy on recurrence patterns in gastric cancer. J Clin Oncol 2010;28:2430-6.

41. McCloskey SA, Yang GY. Benefits and challenges of radiation therapy in gastric cancer: techniques for improving outcomes. Gastrointest Cancer Res 2009;3:15-9.

42. Sun Z, Zhu GL, Lu C, et al. The impact of N-ratio in minimizing stage migration phenomenon in gastric cancer patients with insufficient number or level of lymph node retrieved: results from a Chinese mono-institutional study in 2159 patients. Ann Oncol 2009;20:897-905.

43. Siewert JR, Böttcher K, Stein HJ, et al. Relevant prognostic factors in gastric cancer: ten-year results of the German Gastric Cancer Study. Ann Surg 1998;228:449-61.

44. Marchet A, Mocellin S, Ambrosi A, et al. The prognostic value of $\mathrm{N}$ ratio in patients with gastric cancer: validation in a large, multicenter series. Eur J Surg Oncol 2008;34:159-65.

45. Saito H, Fukumoto Y, Osaki T, et al. Prognostic significance of the ratio between metastatic and dissected lymph nodes (n ratio) in patients with advanced gastric cancer. J Surg Oncol 2008;97:132-5.

46. Wang J, Dang P, Raut CP, et al. Comparison of a lymph node ratiobased staging system with the 7th AJCC system for gastric cancer: analysis of 18,043 patients from the SEER database. Ann Surg 2012;255:478-85.

47. Costa WL, Coimbra FJ, Fogaroli RC, et al. Adjuvant chemoradiotherapy after d2-lymphadenectomy for gastric cancer: the role of n-ratio in patient selection. Results of a single cancer center. Radiat Oncol 2012;7:169. 